\title{
Degenerate Turán problems for hereditary properties
}

\author{
Vladimir Nikiforov \\ Department of Mathematical Sciences, \\ University of Memphis, U.S.A. \\ vnikifrv@memphis.edu \\ Michael Tait* \\ Department of Mathematical Sciences, \\ Carnegie Mellon University, U.S.A. \\ mtait@cmu.edu \\ Craig Timmons ${ }^{\dagger}$ \\ Department of Mathematics and Statistics, \\ California State University Sacramento, U.S.A. \\ craig.timmons@csus.edu
}

Submitted: Jan 27, 2017; Accepted: Nov 7, 2018; Published: Nov 30, 2018

(C) The authors. Released under the CC BY-ND license (International 4.0).

\begin{abstract}
Let $H$ be a graph and $t \geqslant s \geqslant 2$ be integers. We prove that if $G$ is an $n$-vertex graph with no copy of $H$ and no induced copy of $K_{s, t}$, then $\lambda(G)=O\left(n^{1-1 / s}\right)$ where $\lambda(G)$ is the spectral radius of the adjacency matrix of $G$. Our results are motivated by results of Babai, Guiduli, and Nikiforov bounding the maximum spectral radius of a graph with no copy (not necessarily induced) of $K_{s, t}$.
\end{abstract}

Mathematics Subject Classifications: 05C35

\section{Introduction}

Many questions in extremal graph theory start from the classical Turán-type question: given a forbidden subgraph $H$, what is the maximum number of edges in an $n$-vertex graph that does not contain $H$ as a subgraph? This maximum is denoted by $\operatorname{ex}(n, H)$. The Erdös-Stone Theorem gives an asymptotic formula for $\operatorname{ex}(n, H)$ when $\chi(H) \geqslant 3$ which is quadratic in $n$. On the other hand, the Kövari-Sós-Turán Theorem [7] implies that for any bipartite graph $H$, there is a positive constant $\delta$ such that $\operatorname{ex}(n, H)=O\left(n^{2-\delta}\right)$. Turán problems forbidding bipartite graphs are often called degenerate and have been studied extensively [5].

In many cases, theorems in classical extremal graph theory may be strengthened via spectral graph theory. For an $n$-vertex graph $G$, let $\lambda_{1} \geqslant \lambda_{2} \geqslant \cdots \geqslant \lambda_{n}$ be the eigenvalues

*Research supported by National Science Foundation grant DMS-1606350.

$\dagger$ This work was supported by a grant from the Simons Foundation (\#35419, Craig Timmons). 
of its adjacency matrix. Write $\lambda(G)=\lambda_{1}$ for the spectral radius of $G$. Since the spectral radius satisfies the inequality $2 e(G) / n \leqslant \lambda(G)$, any upper bound on $\lambda(G)$ implies an upper bound on $e(G)$. For example, in [10], Nikiforov improved a result of Babai and Guiduli [1] as follows:

Theorem (Nikiforov). Let $s \geqslant t \geqslant 2$, and let $G$ be a $K_{s, t}$ free graph of order $n$. If $t=2$, then

$$
\lambda(G) \leqslant 1 / 2+\sqrt{(s-1)(n-1)+1 / 4} .
$$

If $t \geqslant 3$, then

$$
\lambda(G) \leqslant(s-t+1)^{1 / t} n^{1-1 / t}+(t-1) n^{1-2 / t}+t-2 .
$$

In view of $2 e(G) / n \leqslant \lambda(G)$, the above theorem implies also Füredi's improvement of the Kővari-Sós-Turán Theorem [3].

In this paper, we consider a modification of the Turán-type question, where one forbids induced copies of a subgraph $F$. Without additional restrictions, this problem is trivial if $F$ is not complete, because $K_{n}$ has $\left(\begin{array}{l}n \\ 2\end{array}\right)$ edges and no induced $F$, whereas the problem is thoroughly investigated if $F$ is a complete graph. More precisely, we study the maximum spectral radius that a graph may have if it has no induced $K_{s, t}$ and no copy (not necessarily induced) of a fixed forbidden subgraph $H$. We note that if $\chi(H) \geqslant 3$, then one may forbid $H$ and have quadratically many edges, or one may forbid an induced copy of $K_{s, t}$ and have quadratically many edges. One of the main theorems in [8] shows that when one forbids both $H$ and $K_{s, t}$-induced at the same time, then a graph may not have quadratically many edges.

Theorem (Loh, Tait, Timmons, Zhou [8]). Let $s$ and $t$ be integers and $H$ be a graph. Then there is a constant $C$ depending on $s, t$, and $H$ such that if $G$ is a graph on $n$ vertices which has no copy of $H$ as a subgraph and no copy of $K_{s, t}$ as an induced subgraph, then

$$
e(G)<C n^{2-1 / s} \text {. }
$$

Our main theorems are spectral strengthenings of the above theorem via the same inequality $2 e(G) / n \leqslant \lambda(G)$.

Finally, we note that this problem could be discussed in a more general context. A hereditary graph property is a family of graphs which is closed under isomorphisms and taking induced subgraphs. Given a hereditary property $\mathcal{P}$, let $\mathcal{P}_{n}$ denote the set of $n$ vertex graphs in $\mathcal{P}$. One may ask to find $\operatorname{ex}(n, \mathcal{P}):=\max _{G \in \mathcal{P}_{n}} e(G)$ and $\lambda(n, \mathcal{P}):=$ $\max _{G \in \mathcal{P}_{n}} \lambda(G)$. Nikiforov [11] found the asymptotics of these parameters similarly to the Erdős-Simonovits theorem for monotone graph properties. Let us note that both Erdos-Simonovits's and Nikiforov's theorems are informative only for problems with dense extremal graphs. Not surprisingly, extremal problems leading to sparse extremal graphs are harder and need special methods. As before, we call such problems degenerate.

In this note we focus on a degenerate extremal problem that we feel is quintessential for the area; our main result reads as follows. 
Theorem 1. Let $t \geqslant s \geqslant 3$ be integers, $H$ be a graph, and $K \geqslant\left(R\left(H, K_{t}\right)\right)^{2 / s} R\left(H, K_{s}\right)$. If $G$ is an $H$-free graph of order $n$ and

$$
\lambda(G) \geqslant K n^{1-1 / s}
$$

then $G$ contains an induced copy of $K_{s, t}$.

Here, and throughout the rest of the paper, $R(H, G)$ is the Ramsey number of $H$ vs. $G$. One can question why in the premises of Theorem 1 the parameter $s$ is at least 3 , while 2 seems a more natural value. The reason is that for $s=2$ we can prove a somewhat stronger estimate as stated in the theorem below.

Theorem 2. Let $r \geqslant 2, t \geqslant 2$ be integers, and $K \geqslant R\left(K_{r}, K_{t}\right)$. If $G$ is a $K_{r+1}$-free graph of order $n$ and

$$
\lambda(G) \geqslant K n^{1 / 2},
$$

then $G$ contains an induced copy of $K_{2, t}$.

We note that Theorem 2 may be made more general by forbidding an arbitrary subgraph $H$ instead of $K_{r+1}$. For ease of exposition, we will prove Theorem 2 only when $H=K_{r+1}$. It is clear from the proof of Theorem 1 how to generalize the result. The proofs of the two theorems differ at several points, so we shall keep them separate; they are proved in Section 2.

Finally, in Section 3 we will consider the specific case when $H=C_{5}$ and when an induced copy of $K_{2, t}$ is forbidden. This will serve as an example of how, when more information about $H$ is known, one can obtain close to tight estimates on the multiplicative constant. We will prove the following theorem.

Theorem 3. Let $t \geqslant 2$ be an integer. If $G$ is a $C_{5}$-free graph with no induced copy of $K_{2, t}$, then

$$
\lambda(G) \leqslant \sqrt{t+1+0.375^{1 / 2}} n^{1 / 2}+O\left(n^{3 / 8}\right) .
$$

For each integer $t$ and prime power $q$ for which $t-1$ divides $q^{2}-1$, there is a bipartite $K_{2, t}$-free graph that is $q$-regular and has $\frac{q^{2}-1}{t-1}$ vertices in each part (see Füredi [4]). Such a graph will have spectral radius $\sqrt{\frac{1}{2}(t-1) n+1}$ where $n$ is the number of vertices and so Theorem 3 is best possible up to a multiplicative factor of at most 2 .

Before concluding our introduction, we mention the following corollary to Theorems 1 and 2 which implies one of the main results of [8] mentioned above.

Corollary 4. Let $H$ be a graph and $t \geqslant s \geqslant 2$ be integers. If $G$ is a graph of order $n$ that is $H$-free and has no copy of $K_{s, t}$ as an induced subgraph, then

$$
e(G) \leqslant \frac{1}{2}\left(R\left(H, K_{t}\right)\right)^{2 / s} R\left(H, K_{s}\right) n^{2-1 / s} .
$$




\subsection{Some notation}

If $G$ and $H$ are graphs, we write $H \prec G$ to indicate that $H$ is an induced subgraph of $G$.

Given a graph $G$, we write:

- $V(G)$ for the vertex set of $G$ and $v(G)$ for $|V(G)|$;

- $E(G)$ for the edge set of $G$ and $e(G)$ for $|E(G)|$;

- $\Gamma(X)$ for the set of vertices joined to all vertices of a set $X \subset V(G)$ and $d(X)$ for $|\Gamma(X)|$

- $G[X]$ for the subgraph of $G$ induced by a set $X \subset V(G)$;

- $I_{r}(M)$ for the set of independent $r$-sets of $G$ and $i_{r}(G)$ for $\left|I_{r}(G)\right|$;

- $\lambda(G)$ for the largest eigenvalue of the adjacency matrix of $G$;

- $C_{4}(G)$ for the number of 4-cycles of $G$;

- $\omega(G)$ for the clique number of $G$;

- $k_{r}(G)$ for the number of cliques on $r$ vertices in $G$;

We also write:

- $\left(\begin{array}{c}V \\ r\end{array}\right)$ for the set of $r$-sets of a set $V$;

- $R(H, G)$ for the Ramsey number of $H$ vs. $G$ and $R_{p, q}$ for the Ramsey number of $K_{p}$ vs. $K_{q}$.

\section{Proofs of the main results}

The following technical statement will be used in the proofs of both Theorems 1 and 2 .

Proposition 5. If $G$ is a graph of order $n$, then

$$
\sum_{X \in\left(\begin{array}{c}
V \\
2
\end{array}\right)} d^{2}(X) \geqslant \frac{1}{2}\left(\lambda^{4}(G)-n \lambda^{2}(G)\right) .
$$

Proof. Set for short $V:=V(G)$, and observe that

$$
\sum_{X \in\left(\begin{array}{c}
V \\
2
\end{array}\right)}\left(\begin{array}{c}
d(X) \\
2
\end{array}\right)=2 C_{4}(G) .
$$

Hence,

$$
\begin{aligned}
\sum_{X \in\left(\begin{array}{c}
V \\
2
\end{array}\right)} d^{2}(X) & =4 C_{4}+\sum_{X \in\left(\begin{array}{c}
V \\
2
\end{array}\right)} d(X)=4 C_{4}+\sum_{v \in V}\left(\begin{array}{c}
d(v) \\
2
\end{array}\right) \\
& =4 C_{4}+\frac{1}{2} \sum_{v \in V} d^{2}(v)-e(G) .
\end{aligned}
$$

On the other hand, writing $C W_{4}(G)$ for the number of closed walks of length 4 , it is known that

$$
C W_{4}(G)=8 C_{4}(G)+2 \sum_{i \in V} d^{2}(i)-2 e(G)
$$


Hence inequality (3) implies that

$$
2 \sum_{X \in\left(\begin{array}{l}
V \\
2
\end{array}\right)} d^{2}(X)=8 C_{4}+\sum_{i \in V} d^{2}(i)-2 e(G)=C W_{4}(G)-\sum_{v \in V} d^{2}(v) .
$$

Finally, in view of the identity

$$
C W_{4}(G)=\lambda_{1}^{4}(G)+\cdots+\lambda_{n}^{4}(G)
$$

and Hofmeister's bound

$$
\lambda^{2}(G) \geqslant \frac{1}{n} \sum_{v \in V} d^{2}(v),
$$

inequality (2) follows immediately from (4).

Proof of Theorem 2. Suppose that $t, r, K$, and $G$ satisfy the premises of the theorem. Note that for any pair $X \in\left(\begin{array}{c}V \\ 2\end{array}\right)$, the graph $G[\Gamma(X)]$ is $K_{r}$-free; hence, Turán's theorem implies that

$$
k_{2}(G[\Gamma(X)]) \leqslant \frac{(r-2)}{2(r-1)} d^{2}(X),
$$

and therefore,

$$
i_{2}(G[\Gamma(X)]) \geqslant\left(\begin{array}{c}
d(X) \\
2
\end{array}\right)-\frac{(r-2)}{2(r-1)} d^{2}(X)=\frac{1}{2(r-1)} d^{2}(X)-\frac{1}{2} d(X) .
$$

Summing this inequality over all pairs $X \in\left(\begin{array}{c}V \\ 2\end{array}\right)$ and applying Proposition 5 , we obtain

$$
\begin{aligned}
\sum_{I \in I_{2}(G)}\left(\begin{array}{c}
d(I) \\
2
\end{array}\right) & \geqslant \frac{1}{2(r-1)} \sum_{X \in\left(\begin{array}{c}
V \\
2
\end{array}\right)} d^{2}(X)-\frac{1}{2} \sum_{X \in\left(\begin{array}{l}
V \\
2
\end{array}\right)} d(X) \\
& \geqslant \frac{1}{4(r-1)}\left(\lambda^{4}(G)-n \lambda^{2}(G)\right)-\frac{1}{2} \sum_{v \in V}\left(\begin{array}{c}
d(v) \\
2
\end{array}\right) .
\end{aligned}
$$

Using Hofmeister's bound and some algebra, we find that

$$
\begin{aligned}
\sum_{I \in I_{2}(G)}\left(\begin{array}{c}
d(I) \\
2
\end{array}\right) & \geqslant \frac{1}{4(r-1)}\left(\lambda^{4}(G)-n \lambda^{2}(G)\right)-\frac{1}{4} n \lambda^{2}(G) \\
& =\frac{1}{4(r-1)} \lambda^{2}(G)\left(\lambda^{2}(G)-r n\right) \\
& \geqslant \frac{K^{2}}{4(r-1)}\left(K^{2}-r\right) n^{2} \geqslant \frac{K^{3}}{2}\left(\begin{array}{l}
n \\
2
\end{array}\right)>K\left(\begin{array}{l}
n \\
2
\end{array}\right) .
\end{aligned}
$$

That is to say, there is an $I \in I_{2}(G)$, such that $d(I)>K \geqslant R_{r, t}$. Since $G[\Gamma(I)]$ is $K_{r}$-free, it follows that $\overline{K_{t}} \prec G[\Gamma(I)]$, and so $K_{2, t} \prec G$, completing the proof. 
The proof of Theorem 1 is similar to the proof of Theorem 2, but needs a more technical approach; in particular, Turán's theorem does not apply as above. The focal point of the proof is the fact that if

$$
\sum_{I \in I_{s}(G)}\left(\begin{array}{c}
d(I) \\
2
\end{array}\right) \geqslant\left(\begin{array}{c}
R\left(H, K_{t}\right) \\
2
\end{array}\right)\left(\begin{array}{l}
n \\
s
\end{array}\right),
$$

then $G$ has an independent $s$-set $I$ such that $d(I) \geqslant R\left(H, K_{t}\right)$; since $G[\Gamma(I)]$ is $H$-free, it follows that $\bar{K}_{t} \prec G[\Gamma(I)]$, and hence $K_{s, t} \prec G$.

In turn, we deduce (5) along the following lines: we show that the premises of the theorem imply that $G$ contains many copies of $K_{2, s}$, albeit not necessarily induced. However, the fact that $G$ is $H$-free implies that for a positive proportion of these subgraphs of $G$ their part of size $s$ is an independent set. The requirement $K \geqslant\left(R\left(H, K_{t}\right)\right)^{2 / s} R\left(H, K_{s}\right)$ is sufficient to obtain (5) eventually.

To make this argument precise, we need three additional technical statements.

Proposition 6. If $k \geqslant 2$ and $G$ is a graph of order $n$ with $\lambda(G) \geqslant \sqrt{n}$, then

$$
\sum_{X \in\left(\begin{array}{c}
V \\
2
\end{array}\right)} d^{k}(X) \geqslant \frac{1}{2 n^{k-2}} \lambda^{k}(G)\left(\lambda^{2}(G)-n\right)^{k / 2} .
$$

Proof. The Power Mean inequality and inequality (2) imply that

$$
\begin{aligned}
\left(\left(\begin{array}{l}
n \\
2
\end{array}\right)^{-1} \sum_{X \in\left(\begin{array}{l}
V \\
2
\end{array}\right)} d^{k}(X)\right)^{1 / k} & \geqslant\left(\left(\begin{array}{l}
n \\
2
\end{array}\right)^{-1} \sum_{X \in\left(\begin{array}{l}
V \\
2
\end{array}\right)} d^{2}(X)\right)^{1 / 2} \\
& \geqslant\left(\begin{array}{l}
n \\
2
\end{array}\right)^{-1 / 2}\left(\frac{1}{2}\left(\lambda^{4}(G)-n \lambda^{2}(G)\right)\right)^{1 / 2} .
\end{aligned}
$$

Hence, after simple algebra, we get

$$
\begin{aligned}
\sum_{X \in\left(\begin{array}{c}
V \\
2
\end{array}\right)} d^{2}(X) & \geqslant\left(\begin{array}{c}
n \\
2
\end{array}\right)^{1-k / 2}\left(\frac{1}{2}\left(\lambda^{4}(G)-n \lambda^{2}(G)\right)\right)^{k / 2} \\
& \geqslant n^{2-k} 2^{k / 2-1} 2^{-k / 2}\left(\lambda^{4}(G)-n \lambda^{2}(G)\right)^{k / 2} \\
& =\frac{1}{2 n^{k-2}} \lambda^{k}(G)\left(\lambda^{2}(G)-n\right)^{k / 2} .
\end{aligned}
$$

Proposition 7 . Let $K \geqslant 2, s \geqslant 3$, and $n \geqslant s-1$. If $G$ is a graph of order $n$ with $\lambda(G) \geqslant K n^{1-1 / s}$, then $G$ contains at least

$$
K^{s}\left(\begin{array}{l}
n \\
s
\end{array}\right)
$$

copies of $K_{2, s}$. 
Proof. Proposition 6 implies that

$$
\begin{aligned}
\sum_{X \in\left(\begin{array}{l}
V \\
2
\end{array}\right)} d^{s}(X) & \geqslant \frac{1}{2 n^{s-2}} \lambda^{s}(G)\left(\lambda^{2}(G)-n\right)^{s / 2}>\frac{K^{s} n^{s-1}}{2 n^{s-2}}\left(K^{2} n^{2-2 / s}-n\right)^{s / 2} \\
& \geqslant \frac{K^{s}}{2} n\left(3 n^{2-2 / s}\right)^{s / 2}>2 K^{s} n^{s}
\end{aligned}
$$

Next, we find that

$$
\begin{aligned}
\sum_{X \in\left(\begin{array}{c}
V \\
2
\end{array}\right)}\left(\begin{array}{c}
d(X) \\
s
\end{array}\right) & \geqslant \frac{1}{s !} \sum_{X \in\left(\begin{array}{c}
V \\
2
\end{array}\right)}\left(d^{s}(X)-\left(\begin{array}{l}
s \\
2
\end{array}\right) d^{s-1}(X)\right)=-\frac{\left(\begin{array}{c}
s \\
2
\end{array}\right) n^{s-1}}{s !}+\frac{1}{s !} \sum_{X \in\left(\begin{array}{c}
V \\
2
\end{array}\right)} d^{s}(X) \\
& \geqslant 2 K^{s} \frac{n^{s}}{s !}-\frac{\left(\begin{array}{c}
s \\
2
\end{array}\right) n^{s-1}}{s !} \geqslant K^{s} \frac{n^{s}}{s !}>K^{s}\left(\begin{array}{l}
n \\
s
\end{array}\right) .
\end{aligned}
$$

To complete the proof, it is enough to note that if $s>2$, the sum

$$
\sum_{X \in\left(\begin{array}{l}
V \\
2
\end{array}\right)}\left(\begin{array}{c}
d(X) \\
s
\end{array}\right)
$$

is precisely the number of $K_{2, s}$ copies in $G$.

Proposition 8. Let $s \geqslant 2$. If $G$ is a $H$-free graph of order $n$, then

$$
i_{s}(G) \geqslant\left(\begin{array}{c}
R\left(H, K_{s}\right) \\
s
\end{array}\right)^{-1}\left(\begin{array}{l}
n \\
s
\end{array}\right)-1 .
$$

Proof. If $n<R\left(H, K_{s}\right)$, then the inequality is trivial, so assume $n \geqslant R\left(H, K_{s}\right)$. Since $G$ is $H$-free, any set of $R\left(H, K_{s}\right)$ vertices must contain an independent set of size $s$. Each independent set of size $s$ may be contained in at most

$$
\left(\begin{array}{c}
n-s \\
R\left(H, K_{s}\right)-s
\end{array}\right)
$$

independent sets of size $R\left(H, K_{s}\right)$. Therefore,

$$
i_{s}(G) \geqslant\left(\begin{array}{c}
n \\
R\left(H, K_{s}\right)
\end{array}\right)\left(\begin{array}{c}
n-s \\
R\left(H, K_{s}\right)-s
\end{array}\right)^{-1}=\left(\begin{array}{c}
n \\
s
\end{array}\right)\left(\begin{array}{c}
R\left(H, K_{s}\right) \\
s
\end{array}\right)^{-1} .
$$

Armed with the above propositions, we encounter no difficulty in proving Theorem 1.

Proof of Theorem 1. Suppose that $s, t, H, K$, and $G$ satisfy the premises of the theorem. Note that $n>s-1$, because $n>\lambda(G) \geqslant K n^{1-1 / s}$ and therefore $n>K^{s}>2^{s}>s-1$. Further, for any $X \in\left(\begin{array}{c}V \\ 2\end{array}\right)$, the graph $G[\Gamma(X)]$ is $H$-free; hence, Proposition 8 implies that

$$
i_{s}(G[\Gamma(X)]) \geqslant\left(\begin{array}{c}
R\left(H, K_{s}\right) \\
s
\end{array}\right)^{-1}\left(\begin{array}{c}
d(X) \\
s
\end{array}\right)-1 .
$$


Summing this inequality over all pairs $X \in\left(\begin{array}{l}V \\ 2\end{array}\right)$ and double counting, we obtain

$$
\sum_{I \in I_{s}(G)}\left(\begin{array}{c}
d(I) \\
2
\end{array}\right) \geqslant-\left(\begin{array}{c}
n \\
2
\end{array}\right)+\left(\begin{array}{c}
R\left(H, K_{s}\right) \\
s
\end{array}\right)^{-1} \sum_{X \in\left(\begin{array}{c}
V \\
2
\end{array}\right)}\left(\begin{array}{c}
d(X) \\
s
\end{array}\right) .
$$

On the other hand, Proposition 7 implies that $G$ contains at least

$$
R\left(H, K_{t}\right)^{2} R\left(H, K_{s}\right)^{s}\left(\begin{array}{c}
n \\
s
\end{array}\right)
$$

copies of $K_{2, s}$, that is to say,

$$
\sum_{X \in\left(\begin{array}{c}
V \\
2
\end{array}\right)}\left(\begin{array}{c}
d(X) \\
s
\end{array}\right) \geqslant R\left(H, K_{t}\right)^{2} R\left(H, K_{s}\right)^{s}\left(\begin{array}{l}
n \\
s
\end{array}\right) .
$$

Combining this inequality with (7), we find that

$$
\sum_{I \in I_{s}(G)}\left(\begin{array}{c}
d(I) \\
2
\end{array}\right) \geqslant-\left(\begin{array}{l}
n \\
2
\end{array}\right)+\left(\begin{array}{c}
R\left(H, K_{s}\right) \\
s
\end{array}\right)^{-1} R\left(H, K_{t}\right)^{2} R\left(H, K_{s}\right)^{s}\left(\begin{array}{l}
n \\
s
\end{array}\right)>\left(\begin{array}{c}
R\left(H, K_{t}\right) \\
2
\end{array}\right)\left(\begin{array}{l}
n \\
s
\end{array}\right) .
$$

Therefore, inequality (5) holds; as shown above, it implies Theorem 1.

\section{$3 \quad$ Forbidding $C_{5}$ and induced $K_{2, t}$}

We begin this section with a general lemma that gives an upper bound on $\lambda(G)$ that holds whenever $G$ is $H$-free and has no induced $K_{2, t}$. Because we will be working with eigenvectors, it will be convenient to assume throughout this section that $V(G)=\{1,2, \ldots, n\}$. Furthermore, given a pair of vertices $\{i, j\}$, we will write $d(i, j)$ rather than $d(\{i, j\})$ and we do the same for $\Gamma(\{i, j\})$.

Lemma 9. Let $t \geqslant 2$ be an integer and $H$ be a graph with $h \geqslant 2$ vertices. If $G$ is an $H$-free graph of order $n$ with no induced copy of $K_{2, t}$, then for any vertex $x \in V(H)$,

$$
\lambda(G)^{2} \leqslant\left(R\left(H-x, K_{t}\right)+1\right) n+\left(\sum_{\{i, j\} \in E(G)} d(i, j)^{2}\right)^{1 / 2}\left(\frac{\omega(G)-1}{2 \omega(G)}\right)^{1 / 2} .
$$

Additionally, if $x$ and $y$ is a pair of nonadjacent vertices in $H$, then $R\left(H-x, K_{t}\right)$ can be replaced with $R\left(H-x-y, K_{t}\right)$ in the estimate above.

Proof. Let $x=\left(x_{1}, \ldots, x_{n}\right)$ be a non-negative eigenvector for the eigenvalue $\lambda:=\lambda(G)$ scaled to have 2-norm equal to 1 . We have

$$
\lambda^{2}=\lambda^{2} \sum_{i=1}^{n} x_{i}^{2}=\sum_{i=1}^{n}\left(\lambda x_{i}\right)^{2}=\sum_{i=1}^{n}\left(\sum_{j \in \Gamma(i)} x_{j}\right)^{2}=\sum_{i=1}^{n} d(i) x_{i}^{2}+2 \sum_{1 \leqslant i<j \leqslant n} d(i, j) x_{i} x_{j} .
$$


If $1 \leqslant i<j \leqslant n$ and $\{i, j\} \notin E(G)$, then for any vertex $x \in V(H)$, the common neighborhood $\Gamma(i, j)$ cannot contain a copy of $H-x$ or an independent set of size $t$, otherwise we find a copy of $H$ or an induced copy of $K_{2, t}$. Therefore,

$$
d(i, j)<R\left(H-x, K_{t}\right) .
$$

Using this inequality, we have for any vertex $x \in V(H)$,

$$
\begin{aligned}
\lambda^{2} & =\sum_{i=1}^{n} d(i) x_{i}^{2}+2 \sum_{1 \leqslant i<j \leqslant n} d(i, j) x_{i} x_{j} \\
& <n \sum_{i=1}^{n} x_{i}^{2}+2 \sum_{\{i, j\} \notin E(G)} d(i, j) x_{i} x_{j}+2 \sum_{\{i, j\} \in E(G)} d(i, j) x_{i} x_{j} \\
& <n+2 R\left(H-x, K_{t}\right) \sum_{\{i, j\} \notin E(G)} x_{i} x_{j}+2 \sum_{\{i, j\} \in E(G)} d(i, j) x_{i} x_{j} \\
& \leqslant n+R\left(H-x, K_{t}\right) \sum_{i=1}^{n} \sum_{j=1}^{n} x_{i} x_{j}+2 \sum_{\{i, j\} \in E(G)} d(i, j) x_{i} x_{j} .
\end{aligned}
$$

The double sum

$$
\sum_{i=1}^{n} \sum_{j=1}^{n} x_{i} x_{j}
$$

is at most $n$. This follows from two applications of the Cauchy-Schwarz inequality and the fact that $\|x\|=1$. Therefore,

$$
\lambda^{2} \leqslant\left(1+R\left(H-x, K_{t}\right)\right) n+2 \sum_{\{i, j\} \in E(G)} d(i, j) x_{i} x_{j} .
$$

By Cauchy-Schwarz,

$$
\sum_{\{i, j\} \in E(G)} d(i, j) x_{i} x_{j} \leqslant\left(\sum_{\{i, j\} \in E(G)} d(i, j)^{2}\right)^{1 / 2}\left(\sum_{\{i, j\} \in E(G)} x_{i}^{2} x_{j}^{2}\right)^{1 / 2} .
$$

Since $G$ is $H$-free, $G$ does not contain a complete graph on $h:=|V(H)|$ vertices. As $\sum_{i=1}^{n} x_{i}^{2}=1$, we can apply the Motzkin-Straus inequality [9] to get

$$
\sum_{\{i, j\} \in E(G)} x_{i}^{2} x_{j}^{2} \leqslant \frac{\omega(G)-1}{2 \omega(G)} .
$$

Combining (9) and (10), we have

$$
\sum_{\{i, j\} \in E(G)} d(i, j) x_{i} x_{j} \leqslant\left(\sum_{\{i, j\} \in E(G)} d(i, j)^{2}\right)^{1 / 2}\left(\frac{\omega(G)-1}{2 \omega(G)}\right)^{1 / 2} .
$$


We conclude that for any vertex $x \in V(H)$,

$$
\lambda^{2} \leqslant\left(R\left(H-x, K_{t}\right)+1\right) n+\left(\sum_{\{i, j\} \in E(G)} d(i, j)^{2}\right)^{1 / 2}\left(\frac{\omega(G)-1}{2 \omega(G)}\right)^{1 / 2} .
$$

If $H$ contains a pair of nonadjacent vertices $x$ and $y$, then (8) can be replaced with

$$
d(i, j)<R\left(H-x-y, K_{t}\right)
$$

and the rest of the proof is the same.

We may use Lemma 9 to be more precise than Theorem 2 in the case that we can say something about the number of triangles in the graph.

Proof of Theorem 3. Let $t \geqslant 2$ be an integer and $G$ be a $C_{5}$-free graph with no induced copy of $K_{2, t}$. We must show that

$$
\lambda(G) \leqslant \sqrt{2 t+0.375^{1 / 2}} n^{1 / 2}+O\left(n^{3 / 8}\right) .
$$

Using the fact that $\omega(G) \leqslant 4$ and that a $C_{5}$ minus 2 non-adjacent vertices leaves an edge, we have by Lemma 9 ,

$$
\lambda(G)^{2} \leqslant\left(R\left(K_{2}, K_{t}\right)+1\right) n+\left(\sum_{\{i, j\} \in E(G)} d(i, j)^{2}\right)^{1 / 2}\left(\frac{3}{8}\right)^{1 / 2} .
$$

Note that $R\left(K_{2}, K_{t}\right)=t$. Now

$$
\begin{aligned}
\sum_{\{i, j\} \in E(G)} d(i, j)^{2} & =2 \sum_{\{i, j\} \in E(G)}\left(\begin{array}{c}
d(i, j) \\
2
\end{array}\right)+\sum_{\{i, j\} \in E(G)} d(i, j) \\
& =2 \sum_{\{i, j\} \in E(G)}\left(\begin{array}{c}
d(i, j) \\
2
\end{array}\right)+3 k_{3}(G) \\
& \leqslant 2 \sum_{\{i, j\} \in E(G)}\left(\begin{array}{c}
d(i, j) \\
2
\end{array}\right)+c n^{3 / 2}
\end{aligned}
$$

for some constant $c$. In the last line we have used a result of Bollobas and Györi [2] which bounds the number of triangles in a $C_{5}$-free graph. The sum $\sum_{\{i, j\} \in E(G)}\left(\begin{array}{c}d(i, j) \\ 2\end{array}\right)$ counts pairs of vertices, say $\left\{z_{1}, z_{2}\right\} \subset V(G)$, such that there is an edge $\{i, j\} \in E(G)$ for which $\left\{z_{1}, z_{2}\right\} \subset \Gamma(i, j)$. Suppose that this sum counts the same pair more than once. Let $\left\{z_{1}, z_{2}\right\} \subset \Gamma(i, j) \cap \Gamma(x, y)$. Without loss of generality, we may assume that $i, j$, and $x$ are 
all distinct vertices. In this case, $i j z_{1} x z_{2} i$ is a cycle of length 5 which is a contradiction. Thus,

$$
2 \sum_{\{i, j\} \in E(G)}\left(\begin{array}{c}
d(i, j) \\
2
\end{array}\right) \leqslant 2\left(\begin{array}{l}
n \\
2
\end{array}\right) \leqslant n^{2} .
$$

We conclude that

$$
\lambda(G)^{2} \leqslant(t+1) n+\left(\left(n^{2}+c n^{3 / 2}\right)\right)^{1 / 2}(3 / 8)^{1 / 2} \leqslant\left(t+1+0.375^{1 / 2}\right) n+O\left(n^{3 / 4}\right) .
$$

Taking square roots completes the proof of Theorem 3.

\section{References}

[1] L. Babai, B. Guiduli, Spectral extrema for graphs: the Zarankiewicz problem, Electron. J. Combin. 16 (2009), no. 1, \#R123.

[2] B. Bollobás, E. Györi, Pentagons vs. triangles, Discrete Math. 308 (2008), no. 19, 4332-4336.

[3] Z. Füredi, An upper bound on Zarankiewicz' problem, Combin. Probab. Comput. 5 (1996), no. 1, 29-33.

[4] Z. Füredi, New asymptotics for bipartite Turán numbers, J. Combin. Theory Ser. A 75 (1996), no. 1, 141-144.

[5] Z. Füredi, M. Simonovits, The history of degenerate (bipartite) extremal graph problems, Erdős centennial, Bolyai Soc. Math. Stud., 25, (2013), 169-264.

[6] E. Györi, H. Li, The maximum number of triangles in $C_{2 k+1}$-free graphs, Combin. Probab. Comput. 21 (2012), no. 1-2, 187-191.

[7] T. Kövari, V. Sós, P. Turán, On a problem of K. Zarankiewicz, Colloquium Math. 3 (1954), p. 50-57.

[8] P. Loh, M. Tait, C. Timmons, R. Zhou, Induced Turán numbers, Combinatorics, Probability and Computing, 27 (2018), no. 2, 274-288.

[9] T. S. Motzkin, E. G. Straus, Maxima for graphs and a new proof of a theorem of Turán, Canad. J. Math. 17 (1965), 533-540.

[10] V. Nikiforov, A contribution to the Zarankiewicz problem, Linear Algebra Appl. 432 (2010), no. 6, 1405-1411.

[11] V. Nikiforov, Some extremal problems for hereditary properties of graphs, Electron. J. Combin. 21 (2014), no. 1, \#P1.17. 